\title{
Partial Sums of Some Fractional Operators of Bounded Turning
}

\author{
Zainab E. Abdulnaby* \\ Department of Mathematics, College of Science, Mustansiriyah University, Baghdad, Iraq. \\ E-mail: zainabesa@uomustansiriyah.edu.iq \\ ORCID ID: https://orcid.org/0000-0001-5545-6564
}

Received 16/6/2019, Accepted 29/12/2019, Published 1/12/2020

This work is licensed under a Creative Commons Attribution 4.0 International License.

\begin{abstract}
:
In this paper, several conditions are put in order to compose the sequence of partial sums $\mathfrak{B}_{m}, \mathfrak{L}_{m}$ and $\mathfrak{E}_{m}$ of the fractional operators of analytic univalent functions $\mathfrak{B}^{\mu}, \mathfrak{L}^{v}$ and $\mathfrak{E}_{s, t}$ of bounded turning which are bounded turning too.
\end{abstract}

Key words: Analytic Univalent Functions, Bounded Turning, Fractional Differential Operators, Fractional Integral Operator, Partial Sums.

\section{Introduction:}

In a general series, it is not easy to create a summing of all the preceding terms in the series since the series begins with $n=1$ and moves until infinity. But a geometric series contains many polynomials characteristics, making it handy to work with. In this paper, the way we get the sum of a geometric series is by partial sums. In geometric functions theory, both analytic univalent functions and partial sum polynomials can be used to introduce a new certain linear operator of squares. In addition, the certain values depend on the coefficients in such partial sums.

There are numerous interesting advancements in the partial sums of analytic univalent functions and classes of bounded turning in the open unit disk. In (1) it was proved that the partial sums of the Liberal integral operator of functions of bounded turning are bounded turning too. Moreover, some special functions are associated with calculus operators of analytic univalent functions as in (2) and (3). Recently, the partial

sums of some special functions have been studied by authors for example $(4,5)$ in the domain of open unit disk.

The main results are displayed using the method of partial sums of functions class of bounded turning, we review in this paper some definitions of functions type of bounded turning of analytic univalent and their properties. We begin with a definition of analytic functions and some geometric properties such as convolution (or Hadamard product), that is a binary operation of two or several analytic functions. Also, we consider three types of fractional (differential and integral) operators with their squares of partial sums in the open unit disk.

\section{Preliminaries}

Let $\mathcal{A}$ refer to the family of functions $f$ which are analytic in the domain of the open unit disk $\mathfrak{A}:=\{z ;|z|<1\}$, and is defined by

$$
f(z)=z+\sum_{n=2}^{\infty} a_{n} z^{n}, \quad z \in \mathfrak{A} .
$$

Let the function $f$ be defined in (2.1), then the starlike functions $f$ are functions for which the real part of the quantity $\left(z f^{\prime} / f\right)$ is positive and the convex functions $f$ are functions for which the real part of the quantity $\left(1+z f^{\prime \prime} / f^{\prime}\right)$ is positive. In contrast, the close-to-convex functions $f$ are functions for which the real part of the quantity $\left(f^{\prime} / h^{\prime}, h\right.$ is convex $)$ is positive. The following binary operation $(*)$ symbolizes the convolution (or Hadamard product) of analytic functions in $\mathfrak{A}$ and is defined by

$(f * h)(z)=z+\sum_{n=2}^{\infty} a_{n} b_{n} z^{n}=(h * f)(z)$

where $f(z)$ is defined in (2.1) and $h(z)=z+$ $\sum_{n=2}^{\infty} b_{n} z^{n}$, for $z \in \mathfrak{A}$.

Definition $1(6)$

For $0 \leq \omega<1$, let $f$ in $B(\omega)$ where $B(\omega)$ is a subclass of $\mathcal{A}$, are known as the functions of bounded turning whose derivative contains positive real part and is denoted by

$$
B(\omega):=\left\{f \in \mathcal{A}: \quad \mathfrak{R}\left\{f^{\prime}(z)\right\}>\omega, \quad z \in \mathfrak{A}\right\} .
$$

In (6) the functions in $B(\omega)$ are univalent (oneto-one, or injective) and satified the condition of 
close-to-convex function with $h(z)=z$ in $\mathfrak{A}$. For functions $f$ described in the equation (2.1), many families of analytic and univalent functions are considered (integral and differential) operators type of fractional, which have been introduced by numerous researchers for example (see (7),(8),(9),(10)). Moreover, the familiar fractional calculus operators of analytic and univalent functions $f$ are given in (2.1) which are respectively presented and studied by Srivastava and Owa (11) as follows:

$\mathfrak{B}^{\mu}: \mathcal{A} \rightarrow \mathcal{A}$ for $\mu \notin \mathrm{N} \backslash\{0\}=\{1,2,3, \ldots\}$, is defined by

$$
\begin{aligned}
\mathfrak{B}^{\mu} f(z) & =\Gamma(1-\mu) z^{1+\mu} \mathfrak{D}_{z}^{1+\mu} f(z), \quad z \in \mathfrak{A} \\
& =\sum_{n=0}^{\infty} \frac{\Gamma(1-\mu)}{\Gamma(n+1-\mu)} a_{n+1} z^{n+1} \\
& =z+\sum_{n=2}^{\infty} \frac{\Gamma(1-\mu)}{\Gamma(n-\mu)} a_{n} z^{n}
\end{aligned}
$$

and $\mathfrak{L}^{v}: \mathcal{A} \rightarrow \mathcal{A}$ for $v \notin N \backslash\{0\}=\{1,2,3, \ldots\}$, is defined by

$$
\begin{aligned}
\mathfrak{L}^{v} f(z) & =\Gamma(2-v) z^{v} \mathfrak{D}_{z}^{v} f(z), z \in \mathfrak{A} \\
& =z+\sum_{n=2}^{\infty} \frac{\Gamma(2-v)}{\Gamma(n+1-v)} a_{n} z^{n}
\end{aligned}
$$

where $\mathfrak{D}_{z}^{1+\mu}$ and $\mathfrak{D}_{z}^{v} f(z)$ are respectively in (2.2) and (2.3) the well known fractional differential operators that have been defined by Srivastava and Owa (11), and $\Gamma$ is the Gamma function defined in the form of Factorial function with a positive integer number $q$, such as:

$$
\Gamma(q+1)=q \Gamma(q)=q ! .
$$

Now, a new generation of the fractional integral operator be defined as follows:

$$
\mathfrak{E}_{s, t} f(z)=z+\sum_{n=2}^{\infty} \frac{\Gamma(s)}{\Gamma(t(n-1)+s) Q(\alpha, n)} a_{n} z^{n},
$$

$$
(0 \leq t \leq 1,0<s \leq 1, f \in \mathcal{A}, z \in \mathfrak{R})
$$

where $\mathcal{Q}(\alpha, n)=\left(\begin{array}{c}n+\alpha-1 \\ \alpha\end{array}\right)=\frac{\Gamma(n+\alpha)}{\Gamma(n) \Gamma(\alpha+1)}$, for $\alpha>0$ and $n=\{0,1,2, \ldots\}$ is the binomial coefficient of the fractional integral operator $\mathfrak{E}_{s, t}$.

Observe that the fractional integral operator $\mathfrak{E}_{s, t}$ is generalized for the following operators:

\section{Remark 1}

For $z \in \mathfrak{A}, \mathfrak{R}(t)>0$ and $0<s \leq 1$, we have

1 - if $t=0$ and $s=1$, operator (2.4) gives Noor integral operator (12) and

2- if $\alpha=0$, operator (2.4) gives linear operator defined in (3).

A partial sum is a sum of infinitely several terms at the starting of the geometric series. Next, the $\boldsymbol{m}$-the partial sums of the operators (2.2)-(2.4) are defined as follows:

\section{Definition 2}

For $z \in \mathfrak{A}$, the $\boldsymbol{m}$-the partial sums of the operators in (2.2)-(2.4) are respectively defined by
$\mathfrak{B}_{m}(z)=z+\sum_{n=2}^{m} \frac{\Gamma(1-\mu)}{\Gamma(n-\mu)} a_{n} z^{n}$,

$\mathfrak{L}_{m}(z)=z+\sum_{n=2}^{m} \frac{\Gamma(2-v)}{\Gamma(n+1-v)} a_{n} z^{n}$

and

$\mathfrak{E}_{m}(z)=z+\sum_{n=2}^{m} \frac{\Gamma(s)}{\Gamma(t(n-1)+s) Q(\alpha, n)} a_{n} z^{n}$.

Employing the following lemmas to prove the main results.

\section{Lemma 1 (1)}

For $z \in \mathfrak{U}$, we have

$$
\left\{\sum_{n=1}^{j} \frac{z^{n}}{n+2}\right\}>\frac{-1}{3} \text {. }
$$

\section{Lemma 2 (6)}

Let $p(z) \in \mathfrak{A}$ and $p(z)$ be satisfied $p(0)=1$ where $\mathfrak{R}\{p(z)\}>0.5$ in $\mathfrak{A}$. For $\psi$ analytic function in $\mathfrak{A}$, the values of convolution operation of two analytic functions $(p * \psi)(z)$ are shown in the convex hull (the intersection of all convex set or the smallest convex set) of the image on the domain $\mathfrak{A}$ under construction of function $\psi$.

\section{Results}

By applying Lemma 1 and Lemma 2, we will demonstrate some conditions in order to compose $\boldsymbol{m}$-the partial sums of the operators (2.5), (2.6) and (2.7) of functions of bounded turning that are bounded turning as well.

\section{Theorem 1}

Let $f \in B(\omega)$. If $0.5<\omega<1$ and $0<\mu \leq 0.5$, then we have

$\mathfrak{B}_{m}(z) \in B\left(\frac{3-\Gamma(1-\mu)(1-\omega)}{3}\right), \quad z \in \mathfrak{A}$.

\section{Proof}

Let $f$ be defined in (2.1) besides $f \in B(\omega)$ and $0.5<\omega<1$. Then

$$
\mathfrak{R}\left\{f^{\prime}(z)\right\}>\omega, \quad z \in \mathfrak{A} .
$$

By definition (2.1) leads to,

$\Re\left\{1+\sum_{n=2}^{\infty} n a_{n} z^{n-1}\right\}>\omega>0.5$,

as concerning $0.5<\omega<1$, we obtain

$$
\begin{aligned}
& \mathfrak{R}\left\{1+\sum_{n=2}^{\infty}\left(\frac{n}{1-\omega}\right) a_{n} z^{n-1}\right\}> \\
& \mathfrak{R}\left\{1+\sum_{n=2}^{\infty} n a_{n} z^{n-1}\right\},
\end{aligned}
$$

by appling (3.1) and (3.2), we have

$$
\Re\left\{1+\sum_{n=2}^{\infty}\left(\frac{n}{1-\omega}\right) a_{n} z^{n-1}\right\}>0.5 .
$$

Now, by employing some of the convolution properties to $\mathfrak{B}_{m}^{\prime}(z)$ we have

$$
\begin{aligned}
\mathfrak{B}_{m}^{\prime}(z)= & 1+\sum_{n=2}^{m} \frac{\Gamma(1-\mu)}{\Gamma(n-\mu)} n a_{n} z^{n-1} \\
= & {\left[1+\sum_{n=2}^{m}\left(\frac{n}{1-\omega}\right) a_{n} z^{n-1}\right] * } \\
& \quad\left[1+\sum_{n=2}^{m} \frac{\Gamma(1-\mu)}{\Gamma(n-\mu)}(1-\omega) a_{n} z^{n-1}\right] \\
= & p(z) * Q(z) .
\end{aligned}
$$

As well as from (2.8) and $j=m-1$, we have

$$
\Re\left\{\sum_{n=2}^{m}\left(\frac{1}{n+1}\right) z^{n-1}\right\}>\frac{-1}{3} .
$$

Under the hypothesis 
$\Gamma(n-(\mu+1)+1)=(n-(\mu+1)) !$ and $(n) ! \geq 2^{n}$ for $n \geq 4$, we conclude

$$
(n-(\mu+1)) ! \geq 2^{n-(\mu+3)}, \text { for } n \geq 2
$$

then, for $0<\mu \leq 0.5$ and $n \in N \backslash\{0,1\}$ we obtain the following inquality

$$
\begin{aligned}
& \left|\sum_{n=2}^{m} \frac{z^{n-1}}{\Gamma(n-\mu)}\right|=\left|\sum_{n=2}^{m} \frac{z^{n-1}}{\Gamma(n-(\mu+1)+1)}\right|= \\
& \left|\sum_{n=2}^{m} \frac{z^{n-1}}{(n-(\mu+1)) !}\right| \leq\left|\sum_{n=2}^{m} \frac{z^{n-1}}{2^{n-(\mu+3)}}\right| .
\end{aligned}
$$

Subsequently,

$$
\mathfrak{R}\left\{\sum_{n=2}^{m} \frac{\mathrm{z}^{n-1}}{\Gamma(n-\mu)}\right\} \geq \Re\left\{\sum_{n=2}^{m} \frac{\mathrm{z}^{n-1}}{n+1}\right\},
$$

for $0<\mu \leq 0.5$.

Then by applying Lemma 1, we have

$$
\Re\left\{\sum_{n=2}^{m} \frac{z^{n-1}}{\Gamma(n-\mu)}\right\}>\frac{-1}{3} .
$$

From a simple computation to (3.5) and $Q(z)$ in (3.4) leads to the following

$$
\Re\{Q(z)\}=
$$

$\mathfrak{R}\left\{1+\sum_{n=2}^{m} \frac{\Gamma(1-\mu)}{\Gamma(n-\mu)}(1-\omega) a_{n} z^{n-1}\right\}>$

$$
\frac{3-\Gamma(1-\mu)(1-\omega)}{3},
$$

for $0.5<\omega<1,0<\mu \leq 0.5$ and $|z|<1$.

Moreover,

$$
p(\mathrm{z})=\left(1+\sum_{n=2}^{m}\left(\frac{n}{1-\omega}\right) a_{n} z^{n-1}\right),
$$

where $p(0)=1$ and by applying (11) we have

$\Re\{p(z)\}=\Re\left\{1+\sum_{n=2}^{m}\left(\frac{n}{1-\omega}\right) a_{n} z^{n-1}\right\}>0.5$.

Also by using Lemma 2 , we have shown that

$\mathfrak{R}\left\{\mathcal{B}_{m}^{\prime}(z)\right\}>\frac{3-\Gamma(1-\mu)(1-\omega)}{3}, \quad$ for $z \in \mathfrak{A}$.

\section{Theorem 2}

Let $f \in B(\omega)$ if $0.5<\omega<1$ and $0<v \leq 1$, then we have

\section{Proof}

$$
\mathfrak{L}_{m}(z) \in B\left(\frac{3-\Gamma(2-v)(1-\omega)}{3}\right), \quad z \in \mathfrak{A} .
$$

The prove is similar in the sense to the proof Theorem 1.

\section{Theorem 3}

Let $f \in B(\omega)$ if $0.5<\omega<1$ and $0<s \leq 1$, then we have

\section{Proof}

$$
\mathfrak{E}_{m}(z) \in B\left(\frac{3-\Gamma(s)(1-\omega)}{3}\right), \text { for all }|z|<1 .
$$

From the hypotheses of the theorem, it is well known that

$$
\left\{1+\sum_{n=2}^{\infty} n a_{n} z^{n-1}\right\}>\omega>0.5 \text {, }
$$

for $0.5<\omega<1$, we set

$$
\begin{aligned}
& \Re\left\{1+\sum_{n=2}^{\infty}\left(\frac{n}{1-\omega}\right) a_{n} z^{n-1}\right\}>> \\
& \mathfrak{R}\left\{1+\sum_{n=2}^{\infty} n a_{n} z^{n-1}\right\} .
\end{aligned}
$$

By utilizing (3.6) and (3.7) we have $\mathfrak{R}\left\{1+\sum_{n=2}^{\infty}\left(\frac{n}{1-\omega}\right) a_{n} z^{n-1}\right\}>0.5$ and applying the Hadamard product properties, we obtain

$$
\begin{aligned}
\mathfrak{E}_{m}^{\prime}(z)= & 1+\sum_{n=2}^{m} \frac{n \Gamma(s)}{\Gamma(t(n-1)+s) Q(\alpha, n)} a_{n} z^{n-1} . \\
= & \left(1+\sum_{n=2}^{m}\left(\frac{n}{1-\omega}\right) a_{n} z^{n-1}\right) \times \\
& \left(1+\sum_{n=2}^{m}\left(\frac{(1-\omega) \Gamma(s)}{\Gamma(t(n-1)+s) Q(\alpha, n)}\right) z^{n-1}\right) \\
= & p(z) * \mathcal{W}(z) .
\end{aligned}
$$

Under the hypothesis

$$
\Gamma(n+k) \leq \Gamma(n \beta+k), n, \beta \in N
$$

which is identical to $\frac{1}{\Gamma(n \beta+k)} \leq \frac{1}{\Gamma(n+k)}, n, \beta \in N$ and $n ! \geq 2^{n}$ for $n \geq 4$, we conclude the following inequality

$$
(n+(k-2)) ! \geq 2^{n+(k-4)}, \quad \text { for } n \geq 2
$$

and for $0 \leq t \leq 1$ we obtain

$$
\begin{aligned}
& \left|1+\sum_{n=2}^{m}\left(\frac{\Gamma(s)}{\Gamma(t(n-1)+s)}\right) z^{n-1}\right| \\
& \leq\left|1+\sum_{n=2}^{m}\left(\frac{\Gamma(s)}{\Gamma(n+s-1)}\right) z^{n-1}\right| \\
& =\left|1+\sum_{n=2}^{m}\left(\frac{\Gamma(s)}{(n+s-2) !}\right) z^{n-1}\right| \\
& \quad \leq\left|1+\sum_{n=2}^{m}\left(\frac{\Gamma(s)}{2^{n+s-4}}\right) z^{n-1}\right|
\end{aligned}
$$

from Lemma 1 and

yields

$$
j=m-1, \alpha=0, t \geq 0, s>0
$$

$$
\begin{aligned}
& \mathfrak{R}\left\{1+\sum_{n=2}^{m}\left(\frac{1}{\Gamma(t(n-1)+s)}\right) z^{n-1}\right\} \geq \\
& \mathfrak{R}\left\{1+\sum_{n=2}^{m}\left(\frac{1}{n+1}\right) z^{n-1}\right\}
\end{aligned}
$$

thus

$$
\Re\left\{1+\sum_{n=2}^{m}\left(\frac{1}{\Gamma(t(n-1)+s)}\right) z^{n-1}\right\}>\frac{-1}{3} .
$$

Under the conditions (3.9), we arranged $\mathfrak{R}\{\mathcal{W}(z)\}=\mathfrak{R}\left\{1+\sum_{n=2}^{m}\left(\frac{(1-\omega) \Gamma(s)}{\Gamma(t(n-1)+s)}\right) z^{n-1}\right\}>$ $\frac{3-(1-\omega) \Gamma(s)}{3}$

otherwise,

$p(z)=\left(1+\sum_{n=2}^{m}\left(\frac{n}{1-\omega}\right) a_{n} z^{n-1}\right), \quad z \in \mathfrak{A}$ satisfies: $p(0)=1$, and for $|z|<1$ we have $\mathfrak{R}\{p(z)\}=\left(1+\sum_{n=2}^{m}\left(\frac{n}{1-\omega}\right) a_{n} z^{n-1}\right)>0.5$. By applying Lemma 2 we deduce $\mathfrak{R}\left\{\mathfrak{E}_{m}^{\prime}(z)\right\}>\frac{3-(1-\omega) \Gamma(s)}{3}$, for $(0.5<\omega<1)$ and by this, the proof of Theorem 3 is completed.

\section{Conclusions:}

In this paper, we used the method of partial sums of functions class of bounded turning. The conditions of the partial sums of the fractional (differential and integral) operators are determined to be bounded turning too. 


\section{Acknowledgements:}

The author is thankful to the Mustansiriyah University Baghdad-Iraq for its support in the present work. Also, the author is grateful to the referees for their helpful comments which improve the presentation of the paper.

\section{Author's declaration:}

- Conflicts of Interest: None.

- I hereby confirm that all the Figures and Tables in the manuscript are mine. Besides, the Figures and images, which are not mine, have been given the permission for re-publication attached with the manuscript.

- Ethical Clearance: The project was approved by the local ethical committee in Mustansiriyah University.

\section{References:}

1. Jahangiri JM, Farahmand K. Partial sums of functions of bounded turning. IJMMS. 2003; 4(4): 45-47.

2. Juma AS, Abdulhussain MS, Al-khafaji3 SN. Faber Polynomial Coefficient Estimates for Subclass of Analytic Bi-Bazilevic Functions Defined by Differential Operator. Baghdad Sci J. 2019; 16(1): 248-253.
3. Srivastava HM, Kılıçman A, Abdulnaby ZE, Ibrahim RW. Generalized convolution properties based on the modified Mittag-Leffler function. J Nonlinear Sci Appl. 2017; 10: 4284-4294.

4. Halit O, Yagmur N. Partial sums of generalized Bessel functions. J Math Inequal. 2014; 8: 863-877.

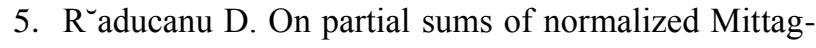
Leffler functions. An St Univ Ovidius Constanta. 2017; 25(2): 123-133.

6. GoodmanAW. Univalent Functions. Washington: Polygonal Publication House; 1983.

7. Abdulnaby ZE, Ibrahim RW, Kılıçman A. Some properties for integro-differential operator defined by a fractional formal. SpringerPlus. 2016; 5(893): 1-9.

8. Esa Z, Srivastava HM, Kılıçman A, Ibrahim RW. A novel subclass of analytic functions specified by a family of fractional derivatives in the complex domain. Filomat. 2017; 31(9): 2837-2849.

9. Arif M, Haq MU, Liu JL. A Subfamily of Univalent Functions Associated with $q$-Analogue of Noor Integral Operator. J Funct Space. 2018; 2018: 1-5.

10. Abdulnaby ZE, Ibrahim RW, Kılıçman A. On boundedness and compactness of a generalized Srivastava-Owa fractional derivative operator. J King Saud Univ Sci. 2018; 30: 1-5.

11. Srivastava HM, Shen CY, Owa S. A linear fractional calculus operator and its applications to certain subclasses of analytic functions. J Math Anal Appl. 1989; 143: 138-147.

12. Noor KI, Noor MA. On integral operators. J Math Anal Appl. 1999; 238: 341-352.

\footnotetext{
الجمع الجزئي لبعض المؤثرات الكسريه ذات الدوال المحدودية

زينب عيسى عبد النبي

قسم الرياضيات، كلية العلوم، ،الجامعة المستتصرية، بغداد، العر اق.

الهذف من هذا البحث هو وضع عدة شروط من اجل تكوين منسلسلة الجمع الجزئي

الدو ال التكافئية التحليلية هن

الكلمات المفتاحية: الدو ال التكافئية التحليلية، الدالة المحدودية، المؤثرات الكسرية التفاضلية، المؤثرات الكسرية التكاملية ,الجمع الجزئي.
} 